\title{
Chapter 4: Principles of statistical analyses: old and new tools
}

Franziska Kretzschmar ${ }^{1,2}$ \& Phillip M. Alday ${ }^{3}$

1 CRC 1252 Prominence in Language, University of Cologne, Cologne, Germany

2 Leibniz-Institute for the German Language, Mannheim, Germany

3 Max Planck Institute for Psycholinguistics, Nijmegen, The Netherlands

running head: principles of statistical analyses

Corresponding author:

Phillip M. Alday

me@phillipalday.com

To appear in:

Grimaldi, M., Y. Shtyrov, \& E. Brattico, (eds.), Language Electrified. Techniques, Methods, Applications, and Future Perspectives in the Neurophysiological Investigation of Language. Springer. 


\begin{abstract}
The present chapter provides an overview of old and new statistical tools to analyze electrophysiological data on language processing. We will first introduce the very basic tenets of experimental designs and their intimate links to statistical design. Based on this, we introduce the analysis of variance (ANOVA) approach which has been the classical statistical tool to analyze event-related potentials (ERPs) in language research. After discussing the merits and disadvantages of the approach, we focus on introducing mixed-effects regression models as a viable alternative to traditional ANOVA analyses. We close with an overview of future directions that mixed-effects modeling opens up for language researchers using ERPs or other electrophysiological measures.
\end{abstract}

Key words: Experimental design, analysis of variance, mixed-effects models, ERPs 


\section{Introduction}

Electrophysiological research on language mainly uses experiments in highly controlled laboratory settings, with the goal of finding systematic relationships between some linguistic manipulation (the independent variable) and participants' brain activity (the dependent variable). Using inferential statistics, we can infer how the systematic relationship between independent and dependent variables in the sample data (with known characteristics) will generalize to the population level (with unknown characteristics).

The hallmark of a good experiment is that it aspires to maintain internal validity given the existing knowledge in the field and the circumstances of data collection (1-4). That is, the experimental design must be set up in a way that allows drawing correct (valid) inferences, by accurately measuring the relationship between the variables we intend to investigate and by doing so reliably. There are two main prerequisites to valid inferences about the relationship between dependent and independent variables: appropriate experimental designs and sampling procedures for internal validity, and appropriate statistical methods that maintain statistical conclusion validity (4).

Statistics are used to separate the signal (the effect of the manipulation) from the noise (the variability inherent in real-world measurements), thereby contributing to valid conclusions about systematic relationships. Statistical conclusions (traditionally expressed in terms of “significance”, see Box “What is statistical significance?”) are often characterized by their potential for Type-I error (detecting a systematic relationship when there is none) and Type-II error (detecting no systematic relationship when there is one), and there is much debate about the best way to keep both types of error at reasonably low levels - and indeed what is even meant by 'reasonably low'. This chapter presents an overview of the key assumptions and statistical tools in analyzing electrophysiological data from neurolinguistic/psycholinguistic experiments, so that statistical conclusion validity - and, consequently, internal validity - can be maintained. 
***Box "What is statistical significance" about here ***

The chapter is organized as follows. We first present those variables that are specific to electroencephalography (EEG) experiments and that may impose further constraints on the type of statistical method (this Section, following subsections). Throughout this chapter, we focus on the analysis of amplitude deflections in event-related potentials (ERPs) for simplicity of presentation. We then go on to discuss the classical way to analyzing electrophysiological data, analysis of variance (ANOVA), also discussing the major disadvantages of ANOVA for EEG/ERP data (Section 2) in language research. In Section 3, we introduce mixed-effects modeling as a viable alternative that can overcome many of these shortcomings, if done carefully. We illustrate the advantage of mixed-effects models with a practical example in Section 4. The chapter closes with an outlook of what avenues research using more sophisticated statistical tools may take in the future (Section 5).

The chapter focus on ANOVA and mixed-effects models is motivated by the observation that the two are the prevailing inferential statistical models - with ANOVA even more so - in previous and current EEG/ERP research on language. Any new researcher will thus likely become familiar with many more ERP results based on these methods compared to other sophisticated statistical tools, the vast majority of which has not been broadly applied in ERPbased language research. To be clear, we do not recommend to restrict one's statistical toolbox to either ANOVA or mixed-effects models (cf. Section 5), but we wish to emphasize their nature and relation to each other - as such an overview chapter is lacking thus far - so that novel researchers know when to apply (or not) which of the two models to their data. Although we focus on the analysis of ERPs from scalp EEG, the analytical methods and considerations described are generally equally applicable to other EEG measures such as peaklatency of ERP components and spectral power in time-frequency representations (TFR) ${ }^{1}$ as 1 We do not discuss the analysis of oscillatory phase as this is a rather specialized topic that does not share much common ground with oscillatory power or time-domain amplitude due to the 'circular' or directional nature of phase, i.e., that the end of one phase is also simultaneously the beginning of another. 
well as magnetoencephalographic (MEG) and intracranial measurements. At times, the content in this chapter will overlap with other chapters in this volume on experimental design, signal processing and basic principles of EEG and its usual interpretation as ERPs or TFRs. This is not by accident: good statistical analysis fundamentally depends on domain-specific knowledge and cannot be viewed as a necessary evil to only be considered after the experiment is done. Likewise, statistical considerations must be included in the experimental design.

\subsection{Experimental design and statistical design}

Figure 1 provides an overview of the steps in electrophysiological research, ranging from initial conceptualization, signal processing and statistics to interpretation.

\section{$* * *$ Figure $1 * * *$}

The experimental design is an operationalization of one's research question and hypotheses, translating them into measurable variables. While there are many experimental designs (2-4), neuro-/psycholinguistics generally uses the factorial design, where researchers test for the influence of one or more independent/experimental variables/factors on one or very few dependent variables. Factorial designs are typically combined with a within-subjects design where subjects contribute observations for more than one condition (i.e., a level of an independent variable/factor). Also, subjects are usually tested on more than one instance for a condition (repeated-measures design implemented as a subjects by trials design, $\boldsymbol{c} f$. 5), i.e., they perceive and respond to several trials with experimental stimuli associated with a condition. This reduces sample size and increases experimental power (i.e., the sensitivity to reveal meaningful changes induced by the independent variables) in comparison to between-subject designs where each participant contributes a single observation for one condition only $(2,5)$. Experimental designs are multi-factorial because of the multidimensionality of EEG as used in neuro-/psycholinguistics: there is both a temporal dimension (the timecourse of the brain response) and a spatial dimension (the location of the electrode on the scalp). So, even if the researcher manipulates only one linguistic independent variable, the multi-dimensional data 
include further variables which will have to be considered in the statistical analysis. This also includes proper treatment of the many sources of confounding variables and random noise in the data, which may obscure the signal and, therefore, need to be addressed with adjustments in experimental design, adequate signal processing procedures or the statistical model. There is no rule of thumb as to which noise source should be treated by which design and analytical procedure, but the general reasoning is that from experimental design to statistical modeling, all steps should converge to "maximize systematic variance, control extraneous variance, and minimize error variance” (6), i.e., control systematic confounds and minimize noise, thereby reducing the total measurement error of the experiment $(2,5)$.

In order to account for the multiple variables, i.e., multiple condition means and various sources of noise, researchers traditionally used Analysis of Variance (ANOVA), an inferential statistical technique for separating variance due to one or more independent variables from other sources. Recently, the field has witnessed a shift to using mixed-effects models to analyze EEG data (7-10), but, to the best of our knowledge, there is as yet little direct comparison of the commonalities and differences of both approaches in EEG/ERP-based language research. The current chapter aims to provide such an overview.

Figure 2 illustrates the typical steps in signal pre-processing, including data export, and the two types of statistical model (ANOVA, mixed-effects regression models [MEM]) that are most commonly performed on electrophysiological measures. ANOVA and mixed-effects models have in common that the variables defined in the experimental design (see Figure 1 above) translate, in statistical terms, into dependent variable (condition means per subject or per trial), experimental variables (also independent variables, factors or predictors) that should induce systematic variance in the data, co-variates associated with unwanted, extraneous variance and blocking variables that represent error variance.

There is an important association between the notions of experimental and blocking variables on the one hand and fixed effects and random effects on the other. Blocking variables are im- 
plemented as random-effect factors in statistical modeling, while experimental variables are usually fixed-effects, but can also represent random-effects factors (cf. Section 3.4 below). A common definition of fixed and random effects is as follows (1, pp. 485-486):

- Fixed effects: The levels of a fixed-effects factor are systematically selected and should typically represent an exhaustive list of factor levels in terms of the research question. Statistical results only generalize to the factor levels included in the design. The (linguistic) manipulations examined by the researcher are implemented as fixed effects.

- Random effects: The levels of a random-effects factor are selected non-systematically or randomly from the population. The sum of levels may not be an exhaustive sample of the respective population. Statistical results generalize to the population, i.e., levels of a random-effects factor can be re-sampled in a replication, without changes in the overall study design. The participants and items in a study are implemented as random effects.

\section{$* * *$ Figure $2 * * *$}

Next, we will present the characteristics of EEG as a method for data collection, highlighting what makes dependent and (some) independent variables in EEG data sets special and why the use of mixed-effects models may consequently be advantageous in analyzing EEG data.

\subsection{The signal: average vs. single-trial}

As discussed in Chapter 5, ERPs are typically computed by averaging the raw EEG across trials, typically within and then across subjects (i.e., a grand average). With enough trials, the non-systematic variability in the EEG cancels out in the averaging procedure, leaving behind only the deflection (the electrical potential) in the EEG synchronized to the time-locking event (event-related). ${ }^{2}$ Note, however, that the ERP is actually present in every trial, just 2 It is important to note here that 'non-systematic' here also includes variability that is not systematic in time. In other words, deflections in the EEG with a variable latency relative to the time-locking event will tend to be 
masked by large variability inherent in EEG: typical language-related effects are on the order of $1-10 \mu \mathrm{V}$ while the spontaneous fluctuations in the EEG are on the order of $10-100 \mu \mathrm{V}$ and muscular artefacts can be on the order of $\mathrm{mV}$, i.e., $1000 \mu \mathrm{V}$. In other words, the averaging procedure serves to separate the signal of interest from everything else, i.e., the signal from the noise. For visual presentation of the ERP, this averaging procedure is very useful. However, for the statistical analysis of the ERP, it is necessary to not just have a measure of the average signal but also of the variability in the signal in order to estimate the balance of signal to noise in the observed deflection. Traditionally in the grand-averaging procedure, the grand-average serves as the observed signal, while the single-subject averages together provide a measure of variability. Unfortunately, this two-stage procedure necessarily destroys information only present at the level of individual trials, such as item-by-item variability.

\subsubsection{Why grand averages and not single-subject averages?}

If the grand-average procedure loses trial-level information, why is a simple averaging procedure across trials not used directly? There are a few reasons for the traditional application of the grand-average procedure for both plotting and statistical purposes, but the core reason is that the grand-average procedure measures the signal and variability across participants instead of across trials and thus the inferences that follow from it are inferences about how the observed effect will generalize to new, unobserved participants instead of new, observed trials. In other words, we can think of the single-subject averages and using multiple observations within each subject as a way to obtain better, less-noisy estimates of the response within that subject. The improved estimates within each subject reduce the noise that each participant introduces, thus enabling us to have better estimates of the variability that arises between subjects. This also follows the logic of repeated-measures ANOVA, which for many decades was the standard analysis procedure for ERP data. In other words, the variability between trials blurred or obscured by the averaging procedure instead of enhanced (see (11) for a more detailed discussion of issues in time-locking). 
(within a given experimental condition) can be coarsely divided into two components: the random variation within each subject and the variation between subjects. We typically do not want to make inferences about how the subjects who took part in the experiment will react to future stimuli, but rather how comparable individuals in the broader population will react to the same stimuli, and thus we use grand averages instead of simple averages.

At this point, there are a few small yet important technical points to emphasize. This twostage procedure applies equally well to first grouping the trials by the types of linguistic stimuli (called items; cf. Section 2.2.1 below) that are used to elicit a response in the participants, instead of by subject, and then computing the grand average across single-item averages. The inference provided by this alternative procedure is then what responses comparable stimuli will elicit from the same participants. In general, these by-subjects and by-items inferences are both desirable and the ideal case would be able to generalize across both items and subjects simultaneously. This challenge is discussed in more detail below (Section 2.2.1). Finally, regardless of whether we are examining inferences across subjects, items, or both, it is useful and desirable to also examine the trial-by-trial variability within each of these grouping units. Doing such single-trial analyses was long considered something exceptional; however, with modern statistical procedures such as mixed-effects models (see Section 3 below), single-trial analyses should no longer be viewed as a cutting-edge or advanced procedure, but rather the current gold standard.

\subsubsection{Grand averages don't stand alone: measures of uncertainty}

Given these considerations about the structuring of variance when examining ERP data, it is somewhat surprising that ERPs are traditionally plotted as grand-average waveforms without any indication about the variability in the underlying data, or equivalently, our uncertainty about the estimated effects that the grand-average represents. While it is considered standard practice to display error bars or confidence intervals on plots and graphs summarizing other 
behavioral measures such as reaction times or response accuracies, it is not common practice to include similar indications of uncertainty in ERP plots. Nonetheless, we strongly recommend that indicators of uncertainty such as error bars or confidence intervals be included in plots of ERP waveforms because they provide crucial information and insight for interpreting whether apparent differences in the waveforms are the result of noise or systematic differences. For display purposes, a by-subjects grand-average with confidence intervals provides a good overview of the effect; however, this is not a replacement for the full statistical procedure for a few reasons. First, it is generally non-trivial with current software to display and compute waveforms and confidence intervals that reflect the full structure of the variability within and across subjects and items. Second, the usual way of computing confidence intervals for the waveforms does not take into account the auto-correlated nature of the ERP timecourse (i.e., that the effect at time $t$ is strongly correlated at time $t-1$ ) and as such they ignore the temporal structure of the data. Similarly, the confidence intervals computed for the timecourse at individual electrodes do not reflect the full spatial structure of the data. Nonetheless, even the coarse impression provided by the grand-average waveform and confidence interval is invaluable in understanding the data.

\subsection{Time-locking in time and space: selecting a region of interest}

EEG data, even when aggregated into amplitude deflections in ERPs, is inherently multidimensional, with each trial containing measurements collected across hundreds of milliseconds and often dozens of electrodes. Most current analysis techniques do not depend on a comprehensive model of the timecourse of the electrophysiological response at every sensor on the scalp, but instead focus on detecting the presence of any systematic difference in the response between two conditions. The naive approach here - to simply test many or even all possible comparisons at different points in time or space - creates a so-called multiple comparisons issue. While traditional statistical approaches provide ways for controlling how often random 
variation is mistaken for a true difference (e.g., Type-I error, see above), there is a subtle yet important point in what often means. In particular, the error rate reflects not the proportion of experiments or research questions in which we expect to arrive at the wrong conclusion, but rather the proportion of statistical tests. Thus, when we perform many tests in order to answer a single question we increase our chances of making an erroneous inference. Given the nominal error rate of 1 in 20 associated with the traditional $p<.05$ significance level and the hundreds of comparisons possible with the multidimensionality of EEG, the naive approach is essentially guaranteed to find an effect even when there is none (see also 12).

Typically, the wealth of data is reduced by coarsening the temporal and spatial information into regions of interests (ROIs), which provide a suitably small set of comparisons. In both dimensions, there are three widely used classes of techniques used to determine appropriate ROIs (12-15):

- a priori definition based on previous research

- visual inspection of independent data or a different comparison

- data-driven approaches that attempt to dynamically determine appropriate ROIs based on the data while nonetheless controlling the Type-I error rate .

A priori methods typically define a certain temporal (e.g., 300-500ms post-stimulus onset for the N400 in adult L1 speakers) or spatial (e.g., the centro-parietal electrodes for the N400 in experiments with rapid serial visual presentation) span and then average across all measurements in that span within a given trial. ${ }^{3}$ These aggregate measurements for a limited number of ROIs are then compared statistically. The advantage to this approach is that it is computationally very simple and fast. The disadvantage is in the reliance on a priori definitions, which requires both clear expectations of when and where an effect will occur (which may not be possible for novel research questions) and may not be 'optimal' for the current dataset (e.g., 3 Given that we tend to simplify in both the temporal and spatial dimensions, the question arises whether we should do the averaging within ROIs within the temporal or spatial dimension first or even within both dimensions simultaneously. Fortuitously, the order actually does not matter due to a mathematical result known as Fubini's Theorem. 
the typical N400 latency across many different experiments will not be exactly the N400 latency associated with a particular manipulation and set of stimuli). As this procedure is not dependent on any aspect of the observed data, it does not increase the Type-I error rate. However, its non-specific approach will tend to decrease power (i.e., increase the Type-II error rate) for any particular experiment where the effects do not exactly match previously observed effects in the literature. However, for experiments where the effects are well predicted by the literature, this procedure will enhance power as there is no need for the types of corrections necessary in more data-driven approaches.

Visual inspection is perhaps the simplest method to perform, but the easiest to use inappropriately. The procedure is quite straightforward: examine the grand-average results from an independent yet related comparison and pick the spatial and temporal ROIs where the effect is at its largest. However, if the same data are used to determine the ROIs as for the statistical comparison, then the inference is circular and invalid (12-14) and known to increase the Type-I error rate. In other words, asking whether there is a difference after adjusting your region of interest to maximize that difference will of course tend to find a difference. The safest way to apply this method is by reserving a subset of the data for determining the ROI and using only the remainder of the data for actual inferences. However, as it is usually not possible to collect a large amount of data for determining the ROI, the usual issues with small sample sizes noisy, unreliable estimates - apply and the resulting ROI may be far from optimal. An alternative comparison is the determination of the overall component structure - as distinct from the effect structure ${ }^{4}$ - by examining the grand-grand mean across all conditions (15). This procedure, although it uses the same data, is nominally not circular because the comparison being drawn for determining the ROIs (time courses of ERP components) is distinct from the comparison being drawn for the inference (where the components differ between conditions). waveform, independent of any comparison, while effects only exist in reference to a particular contrast. For example, visually presented words always elicit an N400, but there is an N400 effect (i.e., an increased N400 amplitude) for unexpected words compared to expected ones. 
Nonetheless, these two comparisons are not fully independent and will thus tend to increase the Type-I error rate. For example, consider the case where condition A elicits a strong P600, but condition B does not elicit this component at all. Then the P600 visible in the grand-grand average depends directly on the difference between conditions. Due to the inherent difficulties in both maintaining independence and reducing variability, we do not generally recommend this approach.

Data-driven approaches on the other hand are often based on a so-called mass-univariate approach, where each temporal and spatial position is tested individually (hence univariate: because the multivariate, i.e., multidimensional nature of the EEG signal is initially ignored) and the results are subsequently adjusted to correct for multiple comparisons (16-20). There are several different methods of performing this correction, each with its own advantages and disadvantages (see 19 for a comparison). An advantage of this data-driven approach, regardless of the particular method, is that it can reveal differences between conditions and does not require strong a priori hypotheses about the temporal and spatial distribution of effects nor an independent testing set. Hence, the approach is particularly useful for studies without a strong hypothesis about the temporal or spatial location of the effect. However, the actual statistical hypothesis being tested by some mass-univariate approaches does not directly test the difference between the emergent ROIs. In particular, the cluster-based approach tests a hypothesis related to the existence of a difference between conditions (more precisely, the exchangeability of the data) and not the location of said difference, even though it uses the clusters of adjacent measurements to reject the null hypothesis of no difference $(\mathbf{1 6 , 1 8 )}$. This leads to the somewhat counter-intuitive fact that the clusters cannot themselves be said to be significant, but instead only help to establish the significance of a non-specific difference between conditions. 


\section{The classical perspective: Analysis of Variance (ANOVA)}

In Section 1.1 above we have argued that the use of analysis of variance (ANOVA) in experimental research on language is motivated by the close tie between experimental design and statistical design. ANOVA designs are implemented so as to statistically assess the impact of sources of systematic variance relative to sources of non-systematic (extraneous or error) variance. The approach has several advantages in comparison to other parametric tests for differences between condition means such as the $t$ test:

1 With ANOVA, one can investigate the influence of more than one independent/experimental variable on the dependent variable, including interactive effects between the experimental variables. In other words, one can compare more than two conditions within the same statistical test.

2 Being an omnibus test for differences between multiple condition means, ANOVA is recommended to constrain whether pairwise comparisons should be performed (21). If a significant effect is found, ANOVA can be complemented with statistical tests for post-hoc pairwise comparisons (where the kind of contrast has not been hypothesized) or for planned a-priori pairwise comparisons (where the kind of contrast has been hypothesized).

3 ANOVA can be performed for one dependent variable (univariate ANOVA) or for multiple dependent variables (multivariate ANOVA).

Importantly, like all statistical tests, ANOVA has a number of assumptions that should be met in order to guarantee the accuracy of the inference based upon it. The following five assumptions apply to between-subject designs where participants provide only one observation for one condition:

1 Normal distribution: The data, when conditioned on the experimental variables, must be normally distributed. (Note that this is not the same as saying that the unconditional, i.e. marginal, data are normally distributed.) 
2 Homogeneity of variance (homoskedacity): The variances within conditions must be equal (homogeneous), otherwise the empirical nominal significance level may diverge from the one chosen by the researcher. This discrepancy also depends on whether there is only one outlier in terms of within-condition variance or whether heterogeneity manifests among several conditions.

3 Scales/levels of measurement: The dependent variable must be metric (interval or ratio scale).

4 Equal sample size: In general, equal sample sizes should contribute to the condition means. Unequal sample sizes are associated with different levels of noise in the variance estimates for different conditions, thereby potentially contributing to violations of the homogeneity of variance. For repeated-measures designs, participants must contribute at least one data point per condition or else they have to be excluded from the analysis.

5 Independence: Observations for the dependent variable must be independent of one another.

Repeated-measures designs where participants provide multiple observations for more than one condition violate the last assumption, because observations from the same subject or triggered by the same items are necessarily correlated with each other. ANOVA for repeatedmeasures designs is therefore subject to a further assumption that is related to the variance homogeneity assumption above.

6 Sphericity: The variances of the differences between pairs of conditions must be equal. Sphericity is at issue when there are at least three conditions to be tested. If these assumptions are violated, ANOVA-based statistics will not follow their nominal error rates, i.e., may yield false positives more often (increased Type-I error) and yet also fail to detect real effects (increased Type-II error). 
Note that violating assumptions does not mean that the error rates will change, just that guarantees about the error rate are no longer valid. There are several studies looking at the practical impacts of violating ANOVA assumptions in practice; however, we will not name them for the following primary reason. The lack of "bad" outcomes in these studies is not a guarantee for similar performance in any other study. Indeed, the point of the ANOVA assumptions is exactly to make guarantees about error rates at the level of individual datasets.

Let us now turn to how ANOVA structures variance to assess the relationship between systematic and non-systematic sources of variance in the data. In what follows, presenting the full mathematical formulae for ANOVA is beyond the scope of the current chapter, but they can be found in every statistics textbook $(\boldsymbol{e . g . ,} \mathbf{1}, \mathbf{2 2})$ where they are covered in sufficient detail. We will focus on the conceptual basis here, with the aim to enable you to assess whether published ERP studies with ANOVA have used the ANOVA procedure adequately.

\subsection{Structuring an ANOVA for Repeated-Measures}

ANOVA aims to describe to what extent the total variance in a data set is determined by systematic variance, induced by fixed effects, and by extraneous and error variance, which are subsumed under non-systematic variance:

total variance $=$ systematic variance + non-systematic variance

It is based on the F-ratio (23) that compares the magnitude of systematic variance with the magnitude of non-systematic variance. Systematic variance means that there are differences in variance between conditions (i.e., the levels of a fixed effect), non-systematic variance means that there are differences within each condition. In other words, the F-ratio evaluates whether the variance associated with the effect is larger the one caused by random factors such as participant or item selection. 
For the F-ratio calculated for repeated-measures designs, the numerator represents variation associated with the effect - that is, the hypothesized differences between two or more condition means -, while the error term in the denominator represents the variance between participants (or items, respectively). If differences in variance between conditions are larger than the differences in variance within each of the conditions, the F-ratio tends to indicate a reliable difference between conditions (assuming a given significance threshold). Hence, the larger the F-ratio when taking the number of conditions and the number of participants (the numerator and denominator degrees of freedom, respectively) into account, the less probable the data are under the assumption of no difference, i.e., the null hypothesis. If this probability is small enough, the null hypothesis (there is no difference between conditions) can be rejected. Violations of the assumptions described in Section 2 above will ultimately cause inaccurate calculation of the F-ratio.

ANOVA is a standard method in all statistical software. For example, in R the typical syntax to analyze mean ERP amplitudes with the built-in function aov() would look like this:

$$
\text { amplitude factor } 1 * \text { factor } 2 * \text { roi + Error(participants/ factor } 1 * \text { factor2*roi) }
$$

The dependent variable is the mean ERP amplitude (in the selected time window of interest). Our linguistic manipulations or fixed effects are modeled with their interaction (factor1*factor2) and with all three interactions with (topographic) ROI (factor1*factor2*roi, factor $1 *$ roi, factor $2 *$ roi). These are the effects we are interested in and that will enter the enumerator in the F-ratio equation. The error term includes a specification of the error units, which are the individual participants. Within-subjects factors, for which participants provide data points for each factor level, are also included in the error-term notation. Between-subjects factors only occur in the notation for the fixed-effects structure.

There are a several important aspects to bear in mind regarding this application of repeatedmeasures ANOVA for ERP studies: 
- There is only a by-participants random effect - specified at the level of the individual participant, because the dependent variable comprises mean amplitude per participant and condition (see also Figure 2).

- Topographic information is included in the fixed effects structure as a categorical factor. There can be one topographic ROI with multiple levels along the sagittal and lateral axes (e.g., four quadrants left and right anterior, left and right posterior plus the vertical midline) or there can be two fixed effects, one per axis. In each case, it is imperative to have an equal number of electrodes per level in order to not violate the assumption of balance for ANOVA.

- The Greenhouse-Geisser or the Huynh-Feldt correction is applied to correct the degrees of freedom that are used to assess the F-ratio for topographic factors, hence addressing sphericity issues (see 22 for further discussion on when to use which correction method).

- For interactions with topographic factors, hierarchical resolution proceeds so that the effect of the linguistic manipulation can be assessed within each level of the topographic ROI factor(s). The resolution of further interactions of non-topographic fixed effects proceeds following one's hypothesis, thereby avoiding resolving interactions more than once.

- Signal preprocessing may lead to data loss in case of excessive artifacts. Participants may have to be excluded if they do not have enough trials in all conditions for averaging. Similarly, by-subject averages are based on possibly very different numbers of trials per condition across participants. As this leads to a lack of balance, participants are often excluded from further analysis if too few trials survive signal processing. There are some major problems with the ANOVA approach that limit the generalizability of the experimental findings and experimental sensitivity. We will turn to them in the next Section. 


\subsection{Problems with classical ANOVAs}

In the following we sketch the main problems that the common practice to analyze ERP data with ANOVA face and that have motivated the use of mixed-effects models. The first two problems (Sections 2.2.1 and 2.2.2) restrict the generalizability of the experimental findings, while the third and fourth problems (Sections 2.2.3 and 2.2.4) also affect the sensitivity to detect effects in how electrophysiological activity changes in response to linguistic input. Indeed, the first two problems - the focus on "significance" instead of estimation and the inability to simultaneously model subject- and item-level variance - motivate us to advocate moving away from ANOVA in language studies and to search for an alternative that addresses these problems (see Section 3 for further discussion).

\subsubsection{Language as a fixed-effect fallacy}

It has been recognized since at least the 1960s that the focus on subject averages leads to the "language as a fixed-effect fallacy” (24-28). Ignoring variance caused by linguistic materials used to elicit language-related responses by participants may induce biases in the analysis and limit generalizability to linguistic material beyond that used in the experiment. At a very basic level item selection is subject to the same considerations that apply to sampling participants, because the item sample should be representative of the infinite population of utterances in a language - just as the participant sample should be representative of the (targeted) speaker population of a language. Consequently, in developing an appropriate ERP design, researchers should not only think of the minimum number of participants and their characteristics, but also on the minimum number of linguistic items and their characteristics (also 29, 30). With this similarity between participant- and item-selection, participants and items have been modeled as random effects or blocking factors in ANOVA analyses in the majority of behavioral studies on language processing (e.g., eye-tracking, self-paced reading or offline judgments). Typically, researchers computed one ANOVA on by-subject averages (F1 analysis) 
and a second ANOVA on by-item averages (F2 analysis) to account for variance induced by random participant characteristics or item characteristics, respectively. However, this has not been the case for ANOVA application on ERP data.

This neglect of accounting for item variance in the data may not only distort the apparent pattern of effects, but it prohibits any generalization to linguistic stimuli other than the ones used in an experiment, thus limiting our inferences and reducing our ability to compare studies with similar manipulations but different stimuli.

\subsubsection{Significance and estimation}

The F-ratio is a comparison of the explained to the unexplained variance, and as such can be interpreted, vaguely, as indicating the strength of the signal. It is common to also report further standardized effect sizes, which more directly measure the actual impact of the manipulation, such as partial eta squared (see 31 for further measures) that provides a measure of the explained variance relative to the total observed variance. While useful in general, there is one main disadvantage. These measures of standardized effect size are indices that reflect how much variance is explained in general by the model inherent in the ANOVA procedure. But they do not provide information of effect size in terms of the measurement units of the dependent variable, that is, in microvolts. If we want to proceed from significance testing to estimating effects and predicting effect size in future (replication) experiments, effect size in terms of microvolts is indispensable.

\subsubsection{Language is both a continuous and a categorical independent variable}

Given its logic, ANOVA requires categorical experimental variables to correctly estimate the amount of variance between and within conditions, which makes it difficult to compute ANOVAs for continuous independent variables. Now, this is unfortunate as language is both continuous and categorical. One strategy researchers have resorted to is the transformation of continuous variables into categorical ones. A case in point here is lexical frequency of words, 
which is inherently continuous, but often enters statistical analysis as a two-level categorical factor, contrasting high-frequency with low-frequency words. Such dichotomization distorts the data, creating a rift between adjacent points near the split, while lumping distant points together into a single group.

\subsubsection{Brain activity is continuous across time and space}

This final point concerns the nature of the dependent variable, the electrophysiological activity itself. The electric field measured by EEG is continuous in both time and space. Yet, with the classical analysis approach introduced in Section 2, ERPs are analyzed with categorical topographic factors, as the typical approach is to group electrode sites into a limited number of spatially distinct ROIs. However, effects are continuous and largely overlap across the scalp. Again, a proper treatment of continuous brain activity pattern eludes the classic ANOVA approach.

\section{A modern perspective: Mixed-effects models}

Mixed-effects models, also called multilevel or hierarchical regression models, present an overarching framework for analyzing electrophysiological (and other) data, subsuming all of 
the considerations discussed thus far. ${ }^{5}$ They present five key advantages over traditional analyses based on significance testing such as ANOVA:

1 They are an explicit regression approach. As such, the estimation of effect sizes is the core of the statistical procedure, in line with modern statistical practice focused on estimation and not statistical significance ( $\boldsymbol{c f}$. 40). These estimates can also serve as tests in their own right, with the careful use of contrast coding (see below) providing an alternative to post-hoc or planned pairwise tests in ANOVA (see also 41). Moreover, regression models are inherently generative in the sense that they can be used to make explicit quantitative predictions about unseen data as well as simulate what future data are expected to look like.

2 They are capable of fitting multiple sources of variance simultaneously (36). This not only resolves the language-as-a-fixed-effect fallacy (Section 2.2.1), but also allows for more refined modeling of other sources of systematic variation in the data. For example, rather than simply 'items', it is possible to model repetition in context sentences vs. the critical word embedded in the target sentence separately. Because all of these sources of repetition can be handled simultaneously, it is not necessary to aver-

5Although the general ideas behind mixed-effects models are found in work in the early to mid $20^{\text {th }}$ century (32-35), they entered the broader psycholinguistic consciousness in 2008 with the publication of a special issue of the Journal of Memory and Language and especially $(36,37)$. The earliest application of mixed-effects models to ERP data that we are aware is from 2009 (38), and, interestingly, it is applied to language data, motivated by the same advantages we espouse above. Two-stage regression techniques (using linear regression within subjects and then performing analysis on the within-subject estimates) start to be seen in ERP studies on language about the same time (39), taking advantage of the richer set of covariates allowed by a regression framework. 
age within subjects or within items to eliminate one source of repetition. In other words, mixed-effects models are well-suited to single-trial analyses.

3 They provide a single technique that can handle a wide range of data types, both in the dependent and the independent variables. In particular for the independent variables, an arbitrary number of both categorical and continuous predictors can be included in the same model. This makes it easier to include covariates and avoid statistical testing of stimulus properties, which is statistically often not justified because the stimuli are a closed set and a difference in properties is less relevant than the effect of any potential difference $(5,42)$. For the dependent variable, the same method can be applied to both metric and non-metric (ordinal, binomial etc.) data with minimal change to the model structure (cf. 37).

4 They are not subject to the same assumptions about balance and symmetry (sphericity) as ANOVA (cf. 36). Beyond eliminating the need for performing tests and correction for sphericity violations, this also makes it possible to include participants for whom only a few trials survived preprocessing. In other words, it is no longer necessary to exclude participants based on trial-survival rates, thus allowing for more data to enter the final analysis. Nonetheless, there is no substitute for a sufficient amount of high quality data. In other words, we still strongly recommend designing the data collection and signal processing to maximize the number of high-quality trials collected from each participant and to collect a sufficient number of participants, as determined, for instance, by a priori power calculations (cf. 43-45).

5 More generally, mixed-effects models are still viable when assumptions are violated because of their generative nature as an explicit regression model. More directly, the observed data can be compared to the predictions from the model to see how well they correspond. Assuming they correspond well, the model can still be interpreted and bootstrapping can be used for estimating robust confidence intervals. Alternatively, the 
model can be used for prediction instead of hypothesis testing (cf. 46 ; this also has parallels to perspectives based in machine learning, which is discussed in Section 5, “Future Directions”, see also 47). Furthermore, the typical method for testing assumptions in ANOVA are additional significance tests, which themselves have assumptions and Type-I and -II error.

Although the general framework for mixed-effects models has been well understood for several decades, the computational requirements (both hardware and software) for regular use have only relatively recently become widely available. In contrast, the computations underlying ANOVA are simple enough that they could in theory be done by hand. However, given the myriad advantages of mixed-effects models and the widespread availability of the necessary computational requirements, we strongly recommend using mixed-effects models and completely deprecating the use of ANOVA.

In the following, we will first discuss how to extend and structure a regression model for repeated measures data (Section 3.1). Building upon that, we will then re-examine and extend the idea of a random effects as introduced for ANOVA, including their misinterpretation as a nuisance parameter (3.2) and the selection of an appropriate random-effects structure (3.3). In light of these considerations for random effects, we then turn our attention to the modeling of topography in mixed-effects models and explain why channel is generally not a random effect

(3.4). Bridging from random effects back to fixed effects, we consider how mixed-effects models adjust the predictions for individual subjects and items by weighting them against the data from all subjects and items (3.5). We conclude by highlighting the importance of contrast coding in interpreting categorical experimental factors.

\subsection{Structuring a Regression Model for Repeated Measures}

The data for a mixed-effects model are processed or prepared exactly the same as in ANOVA with two interrelated exceptions, as can be seen in the left panel of Figure 2. First, the single- 
trial data and not the single-subject averages are used as the dependent variable. Averaging within a spatial or temporal ROI is however performed the same way as for ANOVA but at the single-trial level (see below for additional possibilities for handling ROIs with mixed-effects models). Second, in order to take full advantage of the power of mixed-effects models, it is important that item information for each trial be exported. Traditional EEG processing software did not prioritize the storage nor export of this information because it was lost when averaged within subjects. Moreover, many experimental setups did not log or record item information, although it is generally possible to reconstruct it if the presentation order for each participant is known (i.e., which of several counterbalanced stimuli lists a participant was presented). In other words, some additional advance planning, even at the level of the laboratory setup, may be necessary to guarantee that all the additional information mixed-effects models can take advantage of is recorded.

Mixed-effects models are extensions to the standard linear (and generalized linear) regression models and consist of two parts, the fixed and the random effects. The fixed-effects portion of a mixed-effects model corresponds directly to classical regression and is interpreted as estimates of the "population-level” effects. In other words, the fixed effects correspond to the average effect we would expect for a nonspecific member of the population (of both subjects and items). The random effects correspond to estimates of variance between different group members, such as differences in the effect size between participants. In other words, the random effects capture the structure of the variance due to the repetition within subjects and items. As such, the random effects are sometimes called variance components, e.g. "the subject variance component” describes the portions of the variance that are structured by repetition within subjects.

Both the structure and the terminology for describing random effects are common points of confusion. In order to make the basics a bit clearer before proceeding, let us consider an experiment examining the role of lexical frequency on the N400. In the extended Wilkinson-Ro- 
gers notation (48) used in the popular statistical software R, the basic fixed-effects model would be written as

$$
\text { amplitude } \sim 1+\text { frequency }
$$

where amplitude corresponds to the mean voltage in some a priori chosen time window for the N400. This notation, with the dependent variable on the left of the tilde and the independent variables on the right, corresponds to a basic regression model with one continuous predictor (frequency) and the intercept (indicated here explicitly by the number 1, although R implicitly assumes an intercept term). We could compute this model at each electrode or for each topographic ROI and then combine the models in a second stage as in the mass-univariate approach discussed above. Alternatively, we could include spatial predictors in the model such as:

$$
\text { amplitude } \sim 1+\text { frequency } * \text { roi }
$$

This notation corresponds to a regression model with intercept, two main effects (frequency and roi, where ROI is a categorical predictor corresponding to a set of predefined ROIs) as well as their interaction (allowing for the effect of frequency to differ across the scalp). The model thus far completely ignores the structure of the variance. We begin by modelling the structure induced by the repetition within subjects:

$$
\text { amplitude } \sim 1+\text { frequency * roi }+(1+\text { frequency } * \text { roi } \mid \text { subject })
$$

Here the vertical bar is read as "by" and the parentheses are necessary to demarcate the scope of the structuring. Everything in front of the bar is specified as in the fixed effects. The part after the bar is the grouping variable or more formally the blocking variable. This name already provides a hint as to an important requirement for the variable behind the bar: it must be categorical because it partitions the data and the effects into distinct groups. The portions in front the bar are often called random intercepts (the 1) or random slopes (the two main effects and interaction). In contrast to the fixed effects, the estimates for these random effects will be variances (or equivalently, standard deviations) or the variability in these effects 
between subjects. In other words, the random effects measure an additional level of variability beyond the residual variation that one would expect when measuring real-world data.

A major source of confusion is that the term random effect is sometimes used to indicate the entirety of the random intercepts, slopes and blocking variable and sometimes for any subcomponent thereof. Additionally, the term random is misleading because its technical meaning does not align with its meaning in everyday language. As such, we tend to prefer the term variance components (which also has historical precedent, $\boldsymbol{c f} .49$ ) and then specify the structure of those components by stating the individual structure and blocking variable, e.g. intercepts by subject or by-subject intercepts and slopes for frequency. For the example, we could also include a by-item intercept and slope for ROI:

$$
\text { amplitude } \sim 1+\text { frequency } * \text { roi }+(1+\text { frequency } * \text { roi } \mid \text { subject })+(1+\text { roi } \mid \text { item })
$$

However, a by-item slope for frequency does not make any sense because each item only has a single frequency - the lexical frequency of a word is a static property of that word (at least on the timescales of typical experiments). This is an important point: in some sense, we are fitting regressions within each grouping variable and so it only makes sense to consider random slopes for a blocking variables in which there can reasonably be a slope within each block. With a single static value within each item, there is no by-item slope for frequency. In other words, slopes can only be modeled for effects that are implemented as a within-subjects or within-items factor in the experimental design. In contrast to slopes, which require at least two measurements, intercepts can be estimated without further repetition within the grouping variable. However, if there is no repetition of the grouping variable, e.g. if an item is not repeated across subjects, then this reduces to an observation-level random effect, which is generally inadvisable and unestimable. ${ }^{6}$ Random effects require repetition, both between and within groups.

6 Part of the reason why such a parameter is unestimable is that such a parameter is already in the model in the linear case: the residual variance. More precisely, the residual variance is the variability between observations, which is exactly an observation-level random effect. 
Looking at this model in its entirety, we can begin to consider the interpretation of all the different pieces. The fixed effects work exactly as they would be in classical regression and are statements about the population-level effects. The by-subject random effect has several components. The by-subject intercept reflects variability between subjects in the average N400 amplitude, i.e., it reflects variability between subjects in the quantity measured by the equivalent population-level effect. The by-subject slope for frequency similarly measures the variability in the frequency effect between subjects, while the by-subject slope for ROI captures differences in scalp topography between subjects (which may reflect differences in anatomy, electrophysiology or simply variability in placement of the electrodes). Finally, the by-subject slope for the frequency-by-ROI interaction reflects differences in how the frequency effect interacts with ROI across subjects. For the item effects, the interpretation is similar. The by-item slope reflects a difference in the basic electrophysiological response elicited by a given item, while the by-item slope for ROI reflects differences in topography between items, which may, e.g., reflect differences in the way the representation of each item is distributed across the brain.

\subsection{Misinterpretation as a nuisance variable}

The presentation in Section 3.1 has focused on random effects as capturing the structure in the variance arising naturally from the repetition across and within groups. As such, it should be relatively clear that the grouping variables capture the clustering / grouping of the data, while the random slopes and intercepts capture the variability amongst those groups, i.e., how those groups can differ from each other and from the overall population. This also makes clear why random slopes only make sense for manipulations within a given group: if something only exists between groups, then it does not make sense to discuss how the effect within each group differs from the other groups. The random effects are thus variance components, capturing systematic variation in the population-level effects captured by the fixed effects. The division 
into fixed and random effects thus proceeds on the basis of the grouping inherent in repeated measures (e.g., grouping by participants and by items). Note that this distinction is easier to understand when we break the term "random effect” down into grouping variables and random intercepts and random slopes.

When considering the actual meaning of random effects, i.e., reflecting the actual structure of the data and its variance, we see that factors such as participant should typically be a random effect. Similarly, control variables and covariates such as lexical or acoustic properties of individual stimuli are part of the fixed and not the random effects: they are not part of the clustering of the data, but rather simply covariates. These covariates capture population level effects of potentially uninteresting effects and are thus best described as nuisance parameters, but nuisance parameter is not a synonym for random effects. Indeed, they complement the random effects. For example, lexical covariates capture systematic variation due to certain features or properties, while the random-effects by item capture the systematic variation between items that is not attributable to a particular feature. This holds equally well for by-subject random effects: while demographic variables such as age, gender, education and language background may capture variation between participants, there will generally still be systematic variation between subjects not directly attributable to any particular covariate.

By focusing on random effects as variance components, it also becomes clear why it is necessary to have a sufficient number of groups. In general, it makes little statistical sense to estimate the variance from few observations and the same holds for the variance components; however, the "observations" in such cases are actually the individual groups, i.e., participants or items. A rule of thumb is that at least 6 groups (e.g. 6 test subjects for a by-subject effect) are required to effectively estimate a simple intercept-only random effect. A more general rule of thumb is that roughly 10 observations are required for each parameter to be estimated in a statistical model; fewer than that and the estimates will tend to be very noisy, variable and unreliable. Combining these two perspectives and given that the "observations" for the random 
effects are groups, then we need at least 6-10 additional groups per random slope we wish to estimate. Additionally, the random slopes within a particular blocking variable are not estimated in isolation, instead the correlation between the different random slopes is also estimated. More precisely: both the variance and the covariance of effects within a given blocking variable is estimated. This leads to rapid growth in the number of parameters with each additional random slope. For models with several random slopes this quickly suggests that we need a rather large number of groups. While lme4 and comparable software are capable of fitting models with more parameters in the random effects than levels of the grouping variable, these estimates will tend to be quite noisy and so the question poses itself if such estimates are worthwhile. This point will become critical in the selection of the appropriate random-effects structure (see section below). For small numbers of participants or items, it may instead make more sense to include subject or item as a categorical fixed effect that interacts with the effects of interest. In such cases, the between-groups variability is not estimated, but impact of the grouping is nonetheless controlled for. Note, however, that this approach is only applicable to small number of groups as the resulting number of additional interaction terms quickly becomes prohibitive.

\subsection{Keep it optimal: choosing an appropriate random-effect structure}

Because random effects structure the variance within a model, they also impact the standard errors of the fixed effects. As the $t$-values of the fixed effects are nothing more than the estimate divided by the standard error, and the $t$-values are (either directly or via conversion to $p$ values) often used for significance testing, the choice of random-effects structure has an impact on significance testing. While we strongly encourage researchers to focus on estimation and not significance testing, we nonetheless concede that significance testing will remain part of scientific practice for some time. As such, it is worthwhile to consider the implications of the choice of random effects structure. 
In particular, including a random slope for a particular effect tends to increase the standard error for the corresponding fixed effect. Oversimplifying a bit, the repartitioning of the variance through the random slope makes it clear to the model that the number of independent observations is lower (and it is - observations within a group are not independent; that is what it means to be grouped). As the standard error is inversely proportional to the number of independent observations, this causes the standard error to increase. In other words, the most conservative model (i.e., the model with the lowest Type-I error rate) is the one with maximal possible random effects structure $(\boldsymbol{c} f \cdot \mathbf{5 0 , 5 1 )}$. This has led to the advice to 'keep it maximal' as being a generally accepted rule of thumb. However, this advice quickly breaks down for real designs for both practical and inferential reasons. In practical terms, maximal models are often difficult to fit, with fits often taking a very long time to converge or even failing to converge $(\boldsymbol{c} \boldsymbol{f} . \mathbf{5 0}, \mathbf{5 2}){ }^{7}$ Moreover, as maximal models are often the edge of the feasible parameter space (e.g. with some correlations going to \pm 1 ), many of the usual tests for failed convergence will give false positives, resulting in a situation where the usual diagnostics for model convergence are difficult to assess. ${ }^{8}$ Although there exist a number of possibilities for addressing such difficulties, they are beyond the scope of this brief introduction.

In inferential terms, the number of parameters increases very rapidly with the number of random slopes because a single categorical effect may correspond to several slope parameters (see discussion of contrast coding, below) and each slope parameter adds additional correlation parameters. For example, a full maximal set of random effects for a 2x2x2 design results in 36 parameters (1 intercept, 7 slopes, and 28 correlations) being estimated for each blocking variable. In typical psycholinguistic experiments with about 30 items and 30 participants, this can easily result in more parameters being estimated for a given grouping variable than there are groups. While models with such overparameterized random effects are mathematically 7 Roughly, this means that the software was unable to determine the correct model fit. However, sometimes the software will report a failure to converge even after determining the correct model fit because the software is unable to verify that it had determined the fit.

8 Technically such fits are singular or boundary fits and some diagnostic warnings may use this terminology. 
well-defined and modern software such as lme4 is capable of fitting them, the estimates will tend to be very noisy and not stable (cf. 52-54). More directly: although these models are more conservative in terms of statistical significance, they will tend to be more variable in their estimates and thus more sensitive to noise in the data and tend to make worse predictions because they are overfit. As way of an analogy, such models have started memorizing the data they have seen instead of learning to generalize the underlying pattern.

More recent approaches suggest focusing on selecting parsimonious models that capture the structure of the data but not overfit $(52,53,55)$. Using techniques such as principal component analysis (PCA), it is possible to estimate the effective number of random effects that the data support and use that as a guideline for simplifying model structure $(52,53)$. Hypotheses about the underlying data-generating process, i.e., about where variability is expected to occur, can further guide selection of an appropriate random-effects structure. Moreover, the use of non maximal models does not necessarily inflate Type-I error (55). Finally, as part of the

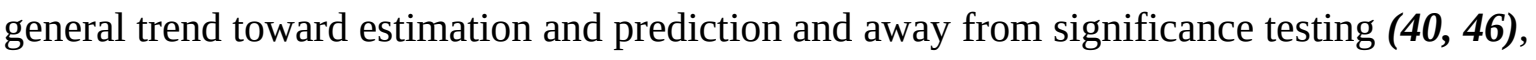
we should not sacrifice estimation and predictive capability out of concerns for statistical significance.

In conclusion, the choice of random effects structure is an area of active debate and research. Our advice is to not blindly apply simple rules such as 'keep it maximal', but rather to take a more nuanced approach based on model diagnostics and hypotheses about where betweengroup variability can reasonably be expected.

\subsection{ROIs revisited: Channel is usually not a random effect}

For EEG recordings, channels present a particular problem. In some sense they represent repeated sampling or grouping in much the same way as participants or items do, which has led some researchers to model channels as a random effect. However, channels display more structured variation than participants or items do because of the correlation between adjacent 
channels. This spatial structure is somewhat problematic for the usual formulation of random effects. In particular, the levels or instances of a grouping variable are usually assumed to be more or less random draws from the set of all possible groups. For example, although a given subject may have certain fixed demographic properties, that subject is generally considered as being chosen at random from the set of all subjects with those properties, such that while the particular offsets (BLUPs) associated with that subject will not be the same as those from a different subject, that subject is nonetheless in some sense interchangeable with a different randomly drawn subject. In other words, while the blocking of the observations into subjects reflects some inherent structure within the data, the choice of any particular subject is not informative. The choice of channel is however informative: this is exactly what we mean when discuss the topography of an effect. This is also why there are by-subjects and by-items ANOVAs, but not by-channel ANOVAs. There are a few straightforward strategies for dealing with this, including two direct extensions of techniques common with ANOVA-based approaches.

The simplest method is simply averaging across channels within a ROI and including the ROI as a fixed effect. As above, this method loses spatial resolution and depends on our ability to define ROIs in advance. However, by categorizing ROIs with two predictors, one for laterality (left vs. right) and one for sagittality (anterior vs. posterior), we directly encode into the model tests of the usual granularity used in describing ERP components. Alternatively, it is possible to apply the data-driven approach: a mixed-effect model is computed for each electrode and then permutation tests are computed using the model instead of a $t$ test or ANOVA (see 27 for an example of this). As mixed-effects models take longer to compute than simple $t$ tests and this approach is already computationally intensive even with $t$ tests, the mass-univariate approach with mixed-effects models can become prohibitively expensive in computational terms. 
In addition to these traditional approaches, mixed-effects models offer an additional alternative for modeling scalp topography without depending on coarse a priori ROIs or computationally expensive permutation or cluster approaches. In particular, instead of modelling discrete electrodes, we can model the continuous distribution of the response across the scalp (as a fixed effect). Each electrode has a location in 3D space, with the x-coordinate corresponding to laterality, the y-coordinate to sagittality and the z-coordinate 'depth' (along the axial dimension running from the top of the head through the spine to the feet). If electrode positions were digitized exactly, then these can be used as the coordinates for each measurement, otherwise the standardized position of the electrodes can be used (as provided by e.g. the international 10-20 system for placing electrodes). An effect for the x-coordinate thus reflects the lateralization of the ERP, while an effect for the y-coordinate indicated a difference between anterior and posterior regions. The z-coordinate is somewhat more difficult to interpret directly; however, experience indicates that including the z-coordinate often improves model fit. One potential interpretation does nonetheless present itself: as more lateral and especially more posterior electrodes tend to lie deeper in the z-dimension than the central electrodes, a significant effect for the z-coordinate may indicate a non-central effect. In all cases, it is worthwhile to plot the model predictions as a topographic plot, in exactly the same way grand-average data can be plotted as scalp topographies. Graphical presentations are often much easier to understand than the textual-numeric model summaries. Finally, while the linearity assumption may seem unreasonable, practical experience suggests that this approach nonetheless performs well. Moreover, the linearity assumption can be relaxed to an assumption of "smooth change”, which will always hold due to the physics of electric fields, by using generalized additive models (GAMs, see Section 4, below).

The advantage to this approach is that does not require a priori definition of ROIs nor the computational overhead of the mass-univariate approach. Moreover, by including all electrodes in a single model, the model can take advantage of the combined information to 
provide better estimates. The disadvantage to this approach is that it does not in its own right give clearly delineated ROIs but instead estimates how the distribution on average changes over the scalp. Moreover, the linearity assumption may be too strong, requiring the application of more advanced methods such as GAMs.

\subsection{Shrinkage and Partial Pooling}

In software such as the R package Ime4 (53), the predictions for how far each level of the grouping differs from the population effect are also available; these are called best linear unbiased predictions (BLUPs) or conditional modes. ${ }^{9}$ The conditional modes correspond to the estimates that a classical regression computed within each subject (or within each item) would have yielded. However, the conditional modes benefit from the extra information available from modelling all of the data simultaneously. In particular, the "inner" regression from each subject and each item informs the regression from every other one in a process called partial pooling (56). This can be thought of as these implicit models borrowing strength from each other and leads to the individual predictions being shrunk toward towards the population mean (57). Individual subjects or items with enough consistency, i.e., enough data points and low within-group variability, will be shrunk less, as they demonstrate strong evidence for a particular pattern, while individual subjects or items with less consistency will be shrunk more towards the population mean. The underlying logic here is essentially "extraordinary claims require extraordinary evidence” and the lack of consistency is taken to indicate a poor estimate of a particular group, in which case that prediction is weighted against the better population-level estimate.

The partial pooling inherent in mixed-effects models is directly responsible for their ability to deal with a lack of balance and allows for the inclusion of subjects where only very few trials survived preprocessing. The extra data from even few trials will help to inform the overall

9 The latter terminology arises because these values correspond to the mode of the distribution of predicted values after taking the grouping variable into account. 
population-level estimate, while the prediction for that subject will take into account the limited evidence for that subject and benefit from the combined evidence across all subjects. Note, however, if many subjects have few trials, then there will still be only very limited data and thus low power. Mixed models do not eliminate the requirement for high-quality data in sufficient amounts.

\subsection{Contrast Coding for fixed effects}

In the ANOVA approach, categorical variables are handled in two steps. During the omnibus F-tests, we are looking for the presence of any difference between levels of a categorical variable, such as between conditions. A significant F-test is then resolved by pairwise t-tests between conditions. In mixed-effects models, categorical variables are instead converted to a set of contrasts, which may represent the difference between two groups, the difference between a group and the grand mean across groups, or any other difference between subdivisions of the data arising from that categorical variable. In particular, contrast coding converts a categorical variable with n categories into a set of n-1 pairwise comparisons. These comparisons can be between two categories, or between one category and the average of several other categories, or between the averages of two different groups of categories. Because these contrasts are encoded directly in the model coefficients, it is not necessary to resolve interactions by fitting simplified models to subsets of the data in a way comparable to resolving Ftests in ANOVA with t-tests.

While it is beyond the scope of this chapter to give a proper tutorial introduction to contrasts (for that see, e.g,. 41), we note that, just as in classical linear regression, the choice of contrasts impacts the interpretation of the model coefficients. More strongly, it is not possible to interpret model coefficients without knowing the contrast coding. While it is possible to specify contrast coding schemes to test particular hypotheses within the data (41), it is still common to use pre-existing coding schemes such as treatment coding and sum coding. As shown 
in Table 1, both of these schemes use pairwise comparisons of a reference level (e.g., a control condition) to all other levels, but they differ in how they encode the reference level, which in turns affects the interpretation of the pairwise comparisons relative to all other variables in the model.

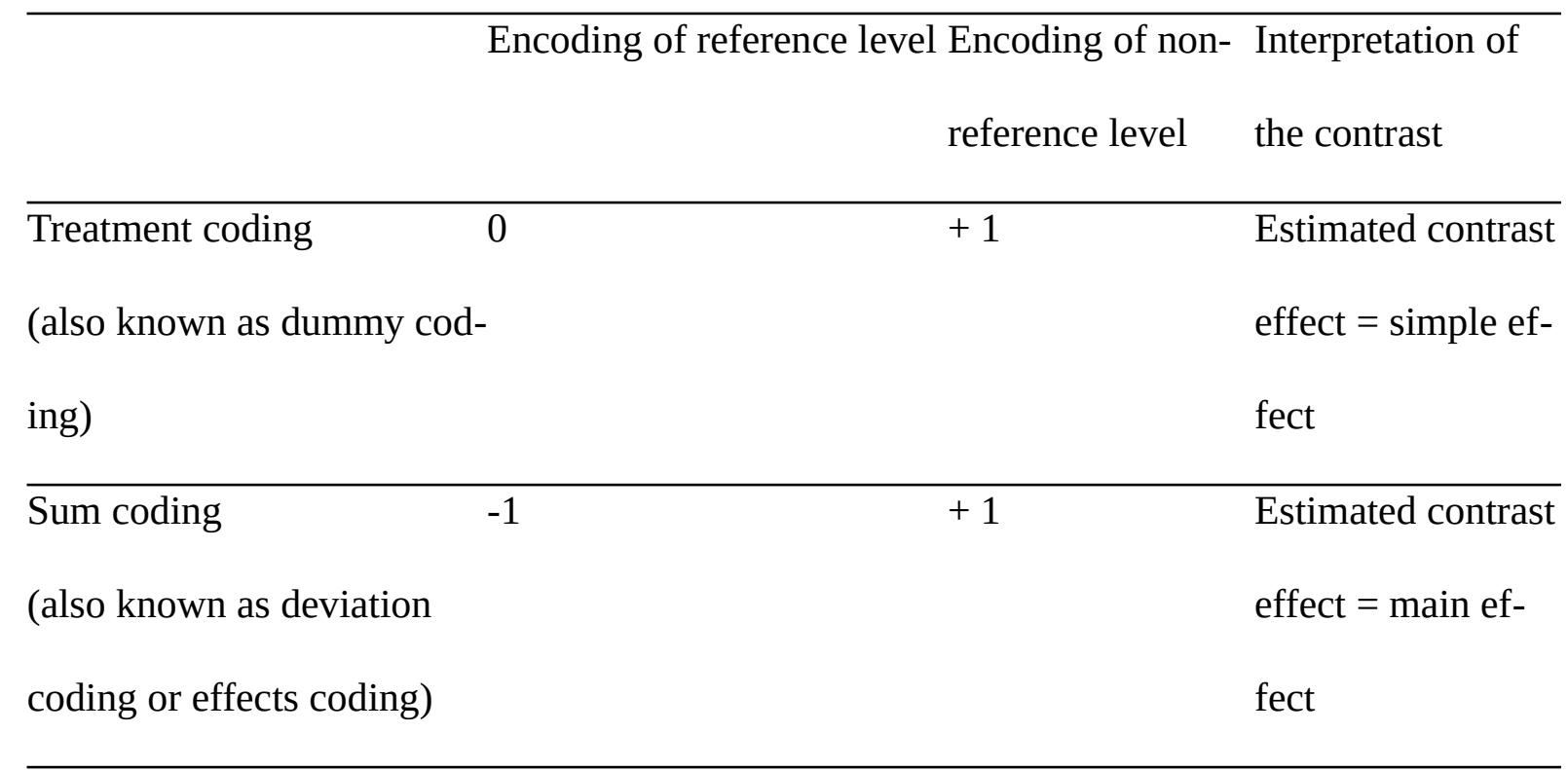

Table 1. Interpretation logic of two pre-existing coding schemes

The effect for a particular contrast reflect the interpretation of a single contrast when all other contrasts are set to zero. In both treatment and sum coding, the non-reference category in a given contrast is represented as +1 . In treatment coding, the reference level is set to 0 , so the effect estimated for a given contrast is the effect of that contrast within the reference level of each of the other variables. This is a simple effect and not properly a main effect, because it is contingent on a specific level of the other contrasts. In sum coding, the reference level is set to -1 , so the effect estimated for a given contrast is the effect of that contrast while ignoring or averaging over the other variables. Ignoring all out all other variables corresponds to setting them to zero and the value zero lies halfway between the top level and the reference level for all the other contrasts.. So setting all other contrasts to zero is the same as setting them to their 
average, and thus sum coding reflects the effect at the average values of all the other predictors, i.e., a main effect.

In other words, sum coding gives the average effect of a categorical variable while not holding otherwise constraining the other variables, while treatment coding gives the effect of a categorical variable while holding the other variables constant at the reference level. As we typically care about these average effects (because as they hierarchically precede simple effects, i.e., reflect effects not conditional on other variables) and not the nested (simple) effects, sum coding is often a sensible choice. Note however that the default coding scheme in $\mathrm{R}$ is treatment coding and so any other coding scheme must be set explicitly. In order to avoid confusion, in both the analysis software and reporting of the results, the contrast coding should always be explicitly stated.

\section{A practical example}

In this section, we illustrate the benefits of analyzing ERP data via mixed-effects models with an empirical example, by using a data set that has previously been analyzed with ANOVA (58) and re-analyzed with mixed-effects models (59). The experimental design is a crossmodal semantic mismatch N400 paradigm, implementing (mis)matches between spoken sentences and visual scene input in a virtual reality environment. As argued in (59), the virtual reality setting presents a particular challenge because of the potential for environmental noise and movement artifacts that may vary from trial to trial.

In the original study, a repeated-measures ANOVA was used to investigate the 2-level linguistic manipulation together with a 5-level topographic ROI and an additional spatial factor for sensors (59 levels, one per scalp electrode). Mean N400 amplitudes were aggregated by participants and condition, i.e., ignoring item and trial variance as is typical in ANOVA analyses of this kind. The results revealed a significant main effect of the linguistic manipulation 
- with larger N400 amplitude for mismatches between the meaning of the sentences and the visual scene - but no interactions with any of the topographic factors.

The virtual reality environment also allows for more variability in the items, yet the traditional F1 (over subject-level averages) analysis does not model this variation. Finally, the authors also reported an exploratory analysis of a later frontal negativity effect.

In (59), these data were reanalyzed using mixed-effects models. The re-analysis used the same time windows and ROIs, but used single-trial data and did not include a factor for channel, instead averaging the data within ROIs. This new analysis yielded two new revelations. First, the item variance was larger than the subject variance, highlighting the importance of modeling both subject and item variance in language studies. Second, when the prestimulus baseline activity was included in the statistical model instead of being subtracted out a priori, the unexpected later effect vanished. Although the conclusions did not fundamentally change in this case - that a large, well-studied and often replicated unimodal effect can also be elicited cross-modally - this study nonetheless highlights how mixed-effects models provide better inferences and are better able to deal with additional sources of variation common in EEG studies of language, such as item variance and baseline drift.

\section{Future directions}

In this chapter, we have presented an overview of the both the classical ANOVA technique and its direct successor, mixed-effects models. In general, both of these techniques are based on treating the EEG as the dependent variable in a linear model. More recently, a number of techniques have been proposed that fundamentally change the structure of how the EEG is modelled: ${ }^{10}$ In the following, we will give a brief overview of their strengths and weaknesses, 10 The specific examples we list here can all be interpreted as variations on the regression framework. More generally, many of the common statistical tools are actually special cases of the general linear model, which is a unifying theory that subsumes much of traditional statistics. 
focusing especially on the two problems that have motivated an increasing number of researchers to move away from ANOVA to mixed-effects models as well as our presentation (i.e., the language-as-a-fixed-fallacy problem and the focus on estimation rather than significance). Given the space limitations of the chapter, this overview can only be a first hint to the interested reader, rather than a comprehensive discussion.

\subsection{Generalization of regression and correlation}

Generalized additive models and generalized additive mixed models (GAMs and GAMMs) do not assume that the relationship between the independent and dependent variables is linear but rather simply smooth. Non-linearity is particularly useful for modeling changes where the difference between steps along a continuum is non-constant because the extremes behave differently, such as lexical frequency effects. In EEG, such effects arise quite naturally as the maximum possible response, i.e., ERP amplitude, is reached (cf. 60 for an example examining N1 amplitude as a function of working memory). GA(M)Ms also allow for modelling dynamics where the change is not monotonic such as adaption effects in an experiment divided into blocks, where the adaption may temporarily decrease at the start of each block (see 61 for an example with a behavioral experiment).

Building on the regression framework from linear and generalized additive models, we can also perform regression at every point along the EEG timecourse and treat the regression coefficients as a timeseries. We can then plot and analyze these regression ERPs (rERPs, 6264). Although this has traditionally been done in a two-stage approach using classical linear regression within subjects $(39,63)$, it is also possible to do this with mixed-effects models, whether linear or generalized additive (see, e.g., 64 and its current development on GitHub for such an extension). When this approach is further extended to include lead and lag predictors, it can also be used for deconvolution, which can control for component overlap and remove certain types of artefacts related to muscle activity such as from eye-movements (cf. 65). 
Several techniques have been proposed as potential methods to analyze EEG data from more naturalistic paradigms, such as auditory stories. Naturalistic paradigms strive to achieve higher ecological validity, but the large temporal variability of language presents a problem for traditional analysis techniques. For example, acoustically red and yellow differ by over 100ms in duration - an eternity in EEG, despite otherwise being rather comparable as similarly frequent color adjectives. As such, traditional techniques with their implicit assumption of equal stimulus duration will not work. A number of alternative analysis techniques have been proposed (see 66 for a review of experimental and statistical solutions), including intersubject correlation (ISC, 67, 68; applied to audiovisual scenes in 69), temporal response functions (TRF, 70, 71), canonical correlation analysis (CCA, 72, 73) and linear convolution (74) Many of these techniques are special applications of the general regression framework. For example, TRFs, rERP and linear convolution can all be formulated as regression problems. The various correlation measures are attempt to find components with the largest correlation between participants (analogous to PCA constructing components with the most shared variance), subject to some constraints. In their usual formulation, however, all of these techniques suffer from the language-as-a-fixed-effect fallacy. For the regression-based techniques, one solution is to use mixed-effects models as the underlying regression technique, but this solution increases the computational cost.

\subsection{Multivariate pattern analysis, machine learning and decoding methods}

In all the techniques thus far, the effect of condition was used to predict the EEG response, i.e., the experimental manipulation was the independent variable and the EEG the dependent variable. However, it is possible to turn this around in a set of techniques adopted from the fMRI literature, namely multivariate pattern analysis (MVPA). The most popular of these techniques attempt to determine the condition the participant was observing from the spatial, temporal or spatio-temporal pattern of the EEG and are thus called decoding techniques as they are often treated as decoding the underlying electrophysiological representation of the 
brain states associated with a particular condition (cf. 75). ${ }^{11}$ Moreover, spatial patterns of activity at one timepoint can be tested against patterns at other points in time. This generalization across time method is useful for characterizing the dynamics of mental representations and be used to test hypotheses related to whether two conditions elicit similar brain activity but at different times (76). Similar to the techniques used for naturalistic stimuli, the usual formulation of decoding methods suffers from the language-as-a-fixed-effect fallacy but this can be addressed. For example, generalization across time often uses logistic regression as the decoder (often called “classifier”, see next paragraph), but there is no fundamental reason why logistic mixed-effects regression cannot be used.

MVPA is often viewed as being fundamentally different from traditional analyses for several reasons. The first is the multivariate nature of these analyses: they fundamentally cannot be performed on a single timepoint or electrode, but instead require the multivariate signal arising from measuring across time and/or space. The second reason is that MVPA analyses often are based on machine learning. Although often viewed as very different, statistics and machine learning are two sides of the same coin (cf. 47$)$, but machine learning tends to focus on prediction without much concern for interpretability of model parameters, while statistics often focuses on inference with a large concern for interpretability of model parameters. This focus on prediction can be a good thing (cf. 46), but it leads to fundamentally different types of statements, such as “decoding performance was above chance”, and the use of a different vocabulary (e.g., “classifier” to describe an algorithm that sorts the data into distinct classes). A full introduction to machine learning, even restricted to ERP, is well beyond the scope of

11 Traditional techniques with the EEG as the response variable are sometimes called encoding techniques in contrast. In encoding techniques, the independent variable encodes the experimental manipulation (i.e., represents it directly), while the independent variable in decoding techniques decodes the experimental manipulation (i.e., extracts it from the dependent variable). 
the chapter, but the machine learning perspective has steadily gained traction across neuroscience in recent years, and so we wish to offer some general advice.

First, interpretability is important in scientific research - the goal of science is after all to understand the world around us better. As such, we find approaches to machine learning that emphasize the interpretation of model parameters (77) very important. Second, although machine learning and statistics are duals of each other, combining the two perspectives can be tricky. For example, many studies use within-subject machine learning analyses based on prediction accuracy and then try to generalize those results to the population using t-tests. This is problematic, both because of the language-as-a-fixed-effect fallacy and because the t-test should not be applied to a bounded value such as an accuracy value (cf. $\mathbf{7 8}$, also for an alternative approach that better captures inter-individual variability). Finally, machine learning methods are often described as “data driven”, which is a misleading description. All machine learning algorithms - like any statistical procedure, even non-parametric ones - have assumptions. It is incumbent upon the user to examine how the violation of those assumptions may influence the estimates and inferences arising from a given analysis. All of these comments hold equally well for machine learning used as an analysis technique or as a language model (e.g., connectionism, distributional semantics, or the recent innovations in using parsers as models of human language comprehension, e.g., 79). Despite these words of caution about machine learning, we are enthusiastic about their addition to the researcher's toolbox (as also evidenced by the second author's work on a software package for decoding analyses, 75).

\subsection{Bayesian and robust approaches}

Orthogonal to all of the considerations and methods we presented thus far (and to each other) are Bayesian statistics and robust statistics. Bayesian statistics have a different perspective on uncertainty than the classical (“frequentist”) approach we used throughout this text. As a massive oversimplification, we can summarize the differences thusly: Bayesian statistics make statements about epistemological uncertainty, while frequentist statistics make state- 
ments about long run frequencies. For example, a Bayesian interpretation of tomorrow’s weather report that there is a $30 \%$ chance of rain reflects the state of his certainty that it will rain tomorrow, while a frequentist would interpret that statement as indicating that it will rain in $30 \%$ of days with the identical weather report. Intuitively, these philosophies are both reasonable and will give the same answers in the long run, but there are important, yet at times subtle differences in the methods and inferences used in the short run. There are a number of good introductions to Bayesian thinking and statistical analysis from a Bayesian perspective, e.g. (80-85).

Robust statistics are statistics that are less sensitive to strict assumptions or the presence of outliers. For example, a single extreme value can lead to the mean being quite far away from most values in the sample. The median meanwhile more accurately represent the center for highly skewed distributions, which is why, for example, median income is more representative of the "typical” income than the mean income when there are even a few exceptionally high earners. Several excellent introduction to robust statistics are available, e.g., (86, 87).For all of the approaches that we can described here - from ANOVA and mixed models to rERPs to decoding approaches -, it is possible to construct a Bayesian or robust or even Bayesian and robust variant, but this may be non trivial, especially in terms of computational cost. Nonetheless, as computational power increases and software implementations continue to improve, we expect Bayesian and robust methods to gain more and more prominence.

\section{Conclusion}

In this chapter, we used ERPs to introduce the common ground of statistical practice in studying the electrophysiology of language. We highlighted that experimental and statistical designs are closely tied to one another. We emphasized the problems with the analysis of variance as a method for language research, despite its use as the classical method in EEG research. We introduced mixed-effects models as a viable modern alternative for language re- 
search and examined the particular challenges that arise when applying this approach to EEG data. By using such methods that are capable of modeling the complexity in inherent in both language and EEG research individually and thus even more so when combined, we are better able to understand this complexity.

\section{References}

1. Keppel G (1991) Design and analysis: A researcher's handbook, 3rd ed. Prentice-Hall, Inc, Englewood Cliffs, NJ, US

2. Kirk RE (2012) Experimental Design. In: Handbook of Psychology, Second Edition. American Cancer Society

3. Abbuhl R, Gass S, Mackey A (2013) Experimental research design. In: Podesva RJ, Sharma D (eds) Research methods in linguistics. Cambridge University Press, Cambridge, pp 116-134

4. Shadish WR, Cook TD, Campbell DT (2001) Experimental and quasi-experimental designs for generalized causal inference. Houghton Mifflin, Boston

5. Myers JL, Well A, Lorch RF (2010) Research design and statistical analysis, 3. ed. Routledge, New York, NY

6. Kerlinger FN (1973) Foundations of behavioral research, 2d ed. Holt, Rinehart and Winston, New York

7. Nieuwland MS, Barr DJ, Bartolozzi F, et al (2020) Dissociable effects of prediction and integration during language comprehension: evidence from a large-scale study using brain potentials. Phil Trans R Soc B 375:20180522. https://doi.org/10.1098/ rstb.2018.0522

8. Roehm D, Sorace A, Bornkessel-Schlesewsky I (2013) Processing flexible form-tomeaning mappings: Evidence for enriched composition as opposed to indeterminacy. Language and Cognitive Processes 28:1244-1274. https://doi.org/ 10.1080/01690965.2012.712143

9. Brennan J, Pylkkänen L (2012) The time-course and spatial distribution of brain activity associated with sentence processing. NeuroImage 60:1139-1148. https://doi.org/ 10.1016/j.neuroimage.2012.01.030

10. Tanner D (2019) Robust neurocognitive individual differences in grammatical agreement processing: A latent variable approach. Cortex 111:210-237. https://doi.org/10.1016/j.cortex.2018.10.011

11. Luck SJ (2014) An introduction to the event-related potential technique, Second edition. The MIT Press, Cambridge, Massachusetts 
12. Luck SJ, Gaspelin N (2017) How to get statistically significant effects in any ERP experiment (and why you shouldn't). Psychophysiology 54:146-157. https://doi.org/10.1111/ psyp.12639

13. Kilner JM (2013) Bias in a common EEG and MEG statistical analysis and how to avoid it. Clinical Neurophysiology 124:2062-2063. https://doi.org/10.1016/ j.clinph.2013.03.024

14. Kriegeskorte N, Simmons WK, Bellgowan PSF, Baker CI (2009) Circular analysis in systems neuroscience: the dangers of double dipping. Nature Neuroscience 12:535-540. https://doi.org/10.1038/nn.2303

15. Brooks JL, Zoumpoulaki A, Bowman H (2017) Data-driven region-of-interest selection without inflating Type I error rate. Psychophysiology 54:100-113. https://doi.org/ 10.1111/psyp.12682

16. Maris E, Oostenveld R (2007) Nonparametric statistical testing of EEG- and MEG-data. Journal of Neuroscience Methods 164:177-190. https://doi.org/10.1016/ j.jneumeth.2007.03.024

17. Pernet CR, Latinus M, Nichols TE, Rousselet GA (2015) Cluster-based computational methods for mass univariate analyses of event-related brain potentials/fields: A simulation study. Journal of Neuroscience Methods 250:85-93. https://doi.org/10.1016/j.jneumeth.2014.08.003

18. Sassenhagen J, Draschkow D (2019) Cluster-based permutation tests of MEG/EEG data do not establish significance of effect latency or location. Psychophysiology 56:e13335. https://doi.org/10.1111/psyp.13335

19. Groppe DM, Urbach TP, Kutas M (2011) Mass univariate analysis of event-related brain potentials/fields I: A critical tutorial review. Psychophysiology 48:1711-1725. https:// doi.org/10.1111/j.1469-8986.2011.01273.x

20. Piai V, Dahlslätt K, Maris E (2015) Statistically comparing EEG/MEG waveforms through successive significant univariate tests: How bad can it be? Psychophysiology 52:440-443. https://doi.org/10.1111/psyp.12335

21. Nieuwenhuis S, Forstmann BU, Wagenmakers E-J (2011) Erroneous analyses of interactions in neuroscience: a problem of significance. Nature Neuroscience 14:1105-1107. https://doi.org/10.1038/nn.2886

22. Field AP, Miles J, Field Z (2012) Discovering statistics using R. Sage, London ; Thousand Oaks, Calif

23. Fisher RA (1925) Statistical methods for research workers. Oliver \& Boyd, Edinburgh

24. Coleman EB (1964) Generalizing to a Language Population. Psychol Rep 14:219-226. https://doi.org/10.2466/pr0.1964.14.1.219

25. Clark HH (1973) The language-as-fixed-effect fallacy: A critique of language statistics in psychological research. Journal of Verbal Learning and Verbal Behavior 12:335-359. https://doi.org/10.1016/S0022-5371(73)80014-3 
26. Judd CM, Westfall J, Kenny DA (2012) Treating stimuli as a random factor in social psychology: A new and comprehensive solution to a pervasive but largely ignored problem. Journal of Personality and Social Psychology 103:54-69. https://doi.org/10.1037/ $\mathrm{a} 0028347$

27. Bürki A, Frossard J, Renaud O (2018) Accounting for stimulus and participant effects in event-related potential analyses to increase the replicability of studies. Journal of Neuroscience Methods 309:218-227. https://doi.org/10.1016/j.jneumeth.2018.09.016

28. Westfall J, Nichols TE, Yarkoni T (2017) Fixing the stimulus-as-fixed-effect fallacy in task fMRI. Wellcome Open Res 1:23. https://doi.org/10.12688/

wellcomeopenres.10298.2

29. Boudewyn MA, Luck SJ, Farrens JL, Kappenman ES (2018) How many trials does it take to get a significant ERP effect? It depends. Psychophysiology 55:e13049. https:// doi.org/10.1111/psyp.13049

30. Judd CM, Westfall J, Kenny DA (2017) Experiments with More Than One Random Factor: Designs, Analytic Models, and Statistical Power. Annu Rev Psychol 68:601-625. https://doi.org/10.1146/annurev-psych-122414-033702

31. Lakens D (2013) Calculating and reporting effect sizes to facilitate cumulative science: a practical primer for t-tests and ANOVAs. Front Psychol 4:. https://doi.org/10.3389/ fpsyg.2013.00863

32. Laird NM, Ware JH (1982) Random-Effects Models for Longitudinal Data. Biometrics 38:963. https://doi.org/10.2307/2529876

33. Fisher RA (1919) XV.- The Correlation between Relatives on the Supposition of Mendelian Inheritance. Trans R Soc Edinb 52:399-433. https://doi.org/10.1017/ S0080456800012163

34. Henderson CR (1973) Sire Evaluation and Genetic Trends. Journal of Animal Science 1973:10-41. https://doi.org/10.1093/ansci/1973.Symposium.10

35. Henderson CR (1982) Analysis of Covariance in the Mixed Model: Higher-Level, Nonhomogeneous, and Random Regressions. Biometrics 38:623. https://doi.org/ $10.2307 / 2530044$

36. Baayen RH, Davidson DJ, Bates DM (2008) Mixed-effects modeling with crossed random effects for subjects and items. Journal of Memory and Language 59:390-412. https://doi.org/10.1016/j.jml.2007.12.005

37. Jaeger TF (2008) Categorical data analysis: Away from ANOVAs (transformation or not) and towards logit mixed models. Journal of Memory and Language 59:434-446. https:// doi.org/10.1016/j.jml.2007.11.007

38. Davidson DJ (2009) Functional Mixed-Effect Models for Electrophysiological Responses. Neurophysiology 41:71-79. https://doi.org/10.1007/s11062-009-9079-y

39. Hauk O, Davis MH, Ford M, et al (2006) The time course of visual word recognition as revealed by linear regression analysis of ERP data. NeuroImage 30:1383-1400. https:// doi.org/10.1016/j.neuroimage.2005.11.048 
40. Cumming G (2014) The New Statistics: Why and How. Psychol Sci 25:7-29. https:// doi.org/10.1177/0956797613504966

41. Schad DJ, Vasishth S, Hohenstein S, Kliegl R (2020) How to capitalize on a priori contrasts in linear (mixed) models: A tutorial. Journal of Memory and Language 110:104038. https://doi.org/10.1016/j.jml.2019.104038

42. Sassenhagen J, Alday PM (2016) A common misapplication of statistical inference: Nuisance control with null-hypothesis significance tests. Brain and Language 162:4245. https://doi.org/10.1016/j.bandl.2016.08.001

43. Westfall J, Kenny DA, Judd CM (2014) Statistical power and optimal design in experiments in which samples of participants respond to samples of stimuli. Journal of Experimental Psychology: General 143:2020-2045. https://doi.org/10.1037/xge0000014

44. Brysbaert M, Stevens M (2018) Power Analysis and Effect Size in Mixed Effects Models: A Tutorial. Journal of Cognition 1:9. https://doi.org/10.5334/joc.10

45. Green P, MacLeod CJ (2016) SIMR : an R package for power analysis of generalized linear mixed models by simulation. Methods Ecol Evol 7:493-498. https://doi.org/ 10.1111/2041-210X.12504

46. Yarkoni T, Westfall J (2017) Choosing Prediction Over Explanation in Psychology: Lessons From Machine Learning. Perspect Psychol Sci 12:1100-1122. https://doi.org/ $10.1177 / 1745691617693393$

47. Breiman L (2001) Statistical Modeling: The Two Cultures (with comments and a rejoinder by the author). Statist Sci 16:199-231. https://doi.org/10.1214/ss/1009213726

48. Wilkinson GN, Rogers CE (1973) Symbolic Description of Factorial Models for Analysis of Variance. Journal of the Royal Statistical Society: Series C (Applied Statistics) 22:392-399. https://doi.org/10.2307/2346786

49. Crump SL (1951) The Present Status of Variance Component Analysis. Biometrics 7:116. https://doi.org/10.2307/3001600

50. Barr DJ, Levy R, Scheepers C, Tily HJ (2013) Random effects structure for confirmatory hypothesis testing: Keep it maximal. Journal of Memory and Language 68:255-278. https://doi.org/10.1016/j.jml.2012.11.001

51. Barr DJ (2013) Random effects structure for testing interactions in linear mixed-effects models. Front Psychol 4:. https://doi.org/10.3389/fpsyg.2013.00328

52. Bates B Douglas M (2019) Complexity in fitting Linear Mixed Models. nextjournal. https://doi.org/10.33016/nextjournal.100002

53. Bates D, Mächler M, Bolker B, Walker S (2015) Fitting Linear Mixed-Effects Models Using lme4. Journal of Statistical Software 67:1-48. https://doi.org/10.18637/ jss.v067.i01

54. Bates D, Kliegl R, Vasishth S, Baayen H (2018) Parsimonious Mixed Models. arXiv:150604967 [stat] 
55. Matuschek H, Kliegl R, Vasishth S, et al (2017) Balancing Type I error and power in linear mixed models. Journal of Memory and Language 94:305-315. https://doi.org/ 10.1016/j.jml.2017.01.001

56. Gelman A, Hill J (2007) Data analysis using regression and multilevel/hierarchical models. Cambridge University Press, Cambridge ; New York

57. Pinheiro JC, Bates DM (2000) Mixed-Effects Models in S and S-PLUS. Springer-Verlag, New York

58. Tromp J, Peeters D, Meyer AS, Hagoort P (2018) The combined use of virtual reality and EEG to study language processing in naturalistic environments. Behav Res 50:862869. https://doi.org/10.3758/s13428-017-0911-9

59. Alday PM (2019) How much baseline correction do we need in ERP research? Extended GLM model can replace baseline correction while lifting its limits. Psychophysiology 56:. https://doi.org/10.1111/psyp.13451

60. Tremblay A, Newman AJ (2015) Modeling nonlinear relationships in ERP data using mixed-effects regression with R examples. Psychophysiology 52:124-139. https:// doi.org/10.1111/psyp.12299

61. Baayen H, Vasishth S, Kliegl R, Bates D (2017) The cave of shadows: Addressing the human factor with generalized additive mixed models. Journal of Memory and Language 94:206-234. https://doi.org/10.1016/j.jml.2016.11.006

62. Smith NJ, Kutas M (2015) Regression-based estimation of ERP waveforms: I. The rERP framework. Psychophysiology 52:157-168. https://doi.org/10.1111/psyp.12317

63. Smith NJ, Kutas M (2015) Regression-based estimation of ERP waveforms: II. Nonlinear effects, overlap correction, and practical considerations. Psychophysiology 52:169181. https://doi.org/10.1111/psyp.12320

64. Ehinger BV, Dimigen O (2019) Unfold: an integrated toolbox for overlap correction, non-linear modeling, and regression-based EEG analysis. PeerJ 7:e7838. https://doi.org/ 10.7717/peerj.7838

65. Dimigen O, Ehinger BV (2019) Analyzing combined eye-tracking/EEG experiments with (non)linear deconvolution models. Neuroscience

66. Alday PM (2019) M/EEG analysis of naturalistic stories: a review from speech to language processing. Language, Cognition and Neuroscience 34:457-473. https://doi.org/ $10.1080 / 23273798.2018 .1546882$

67. Hasson U (2004) Intersubject Synchronization of Cortical Activity During Natural Vision. Science 303:1634-1640. https://doi.org/10.1126/science.1089506

68. Lankinen K, Saari J, Hari R, Koskinen M (2014) Intersubject consistency of cortical MEG signals during movie viewing. NeuroImage 92:217-224. https://doi.org/10.1016/ j.neuroimage.2014.02.004 
69. Cohen SS, Parra LC (2016) Memorable Audiovisual Narratives Synchronize Sensory and Supramodal Neural Responses. eNeuro 3:ENEURO.0203-16.2016. https://doi.org/ 10.1523/ENEURO.0203-16.2016

70. Ding N, Simon JZ (2012) Emergence of neural encoding of auditory objects while listening to competing speakers. Proceedings of the National Academy of Sciences 109:11854-11859. https://doi.org/10.1073/pnas.1205381109

71. Broderick MP, Anderson AJ, Di Liberto GM, et al (2018) Electrophysiological Correlates of Semantic Dissimilarity Reflect the Comprehension of Natural, Narrative Speech. Current Biology 28:803-809.e3. https://doi.org/10.1016/j.cub.2018.01.080

72. Arana S, Marquand A, Hultén A, et al (2020) Sensory Modality-Independent Activation of the Brain Network for Language. J Neurosci 40:2914-2924. https://doi.org/10.1523/ JNEUROSCI.2271-19.2020

73. Zhang W, Ding N (2017) Time-domain analysis of neural tracking of hierarchical linguistic structures. NeuroImage 146:333-340. https://doi.org/10.1016/ j.neuroimage.2016.11.016

74. Brodbeck C, Presacco A, Simon JZ (2018) Neural source dynamics of brain responses to continuous stimuli: Speech processing from acoustics to comprehension. NeuroImage 172:162-174. https://doi.org/10.1016/j.neuroimage.2018.01.042

75. Bode S, Feuerriegel D, Bennett D, Alday PM (2019) The Decision Decoding ToolBOX (DDTBOX) - A Multivariate Pattern Analysis Toolbox for Event-Related Potentials. Neuroinform 17:27-42. https://doi.org/10.1007/s12021-018-9375-z

76. King J-R, Dehaene S (2014) Characterizing the dynamics of mental representations: the temporal generalization method. Trends in Cognitive Sciences 18:203-210. https:// doi.org/10.1016/j.tics.2014.01.002

77. Haufe S, Meinecke F, Görgen K, et al (2014) On the interpretation of weight vectors of linear models in multivariate neuroimaging. NeuroImage 87:96-110. https://doi.org/ 10.1016/j.neuroimage.2013.10.067

78. Allefeld C, Görgen K, Haynes J-D (2016) Valid population inference for informationbased imaging: From the second-level t -test to prevalence inference. NeuroImage 141:378-392. https://doi.org/10.1016/j.neuroimage.2016.07.040

79. Hale J, Dyer C, Kuncoro A, Brennan J (2018) Finding syntax in human encephalography with beam search. In: Proceedings of the 56th Annual Meeting of the Association for Computational Linguistics (Volume 1: Long Papers). Association for Computational Linguistics, Melbourne, Australia, pp 2727-2736

80. Etz A, Vandekerckhove J (2018) Introduction to Bayesian Inference for Psychology. Psychon Bull Rev 25:5-34. https://doi.org/10.3758/s13423-017-1262-3

81. Sorensen T, Hohenstein S, Vasishth S (2016) Bayesian linear mixed models using Stan: A tutorial for psychologists, linguists, and cognitive scientists. TQMP 12:175-200. https://doi.org/10.20982/tqmp.12.3.p175 
82. Schad DJ, Betancourt M, Vasishth S (2020) Toward a principled Bayesian workflow in cognitive science. Psychological Methods. https://doi.org/10.1037/met0000275

83. Kruschke JK (2010) What to believe: Bayesian methods for data analysis. Trends in Cognitive Sciences 14:293-300. https://doi.org/10.1016/j.tics.2010.05.001

84. Kruschke JK, Liddell TM (2018) Bayesian data analysis for newcomers. Psychon Bull Rev 25:155-177. https://doi.org/10.3758/s13423-017-1272-1

85. Kruschke JK, Liddell TM (2018) The Bayesian New Statistics: Hypothesis testing, estimation, meta-analysis, and power analysis from a Bayesian perspective. Psychon Bull Rev 25:178-206. https://doi.org/10.3758/s13423-016-1221-4

86. Wilcox RR (2010) Fundamentals of Modern Statistical Methods. Springer New York, New York, NY

87. Wilcox RR (2012) Introduction to robust estimation and hypothesis testing, 3rd ed. Academic Press, Amsterdam ; Boston

88. Cohen J (1992) Things I have learned (so far). In: Kazdin AE (ed) Methodological issues \& strategies in clinical research. American Psychological Association, Washington, pp 315-333

89. Cohen J (1994) The earth is round ( $\mathrm{p}<.05)$. American Psychologist 49:997-1003. https://doi.org/10.1037/0003-066X.49.12.997 


\section{BOX 1}

\section{What is statistical significance? What are $p$ -}

\section{values?}

Classical statistical inference is performed on the basis of null-hypothesis significance testing. In this framework, the null hypothesis, typically that the experimental manipulation had no effect and that any observed differences are purely due to chance, is first assumed to be true. We quantify the observed differences by means of a test statistic such as the F-value (the ratio of variances between and within test groups) or the t-value (the ratio of estimate to estimated error). Under this assumption, it is possible to estimate the probability of observing a test statistic at least as large as the observed one; this probability is called the p-value. If this probability is sufficiently low (traditionally less than 5\%, i.e., $\mathrm{p}<0.05$ ), then the difference is called significant and the null hypothesis is rejected as being an unreasonable assumption. Usually this is taken as sufficient ground to accept the actual hypothesis of interest, but note that the logic here is quite indirect and based on probabilistic and not deterministic statements (88, 89). In particular, the p-value tells us nothing about the probability that the null hypothesis is true nor that the research hypothesis is true, as it is a probability computed under the assumption that the null hypothesis holds. Furthermore, it is important to note that statistical significance says nothing about practical significance and that the p-value says nothing about the actual size of the effect. 


\section{Figure 1}

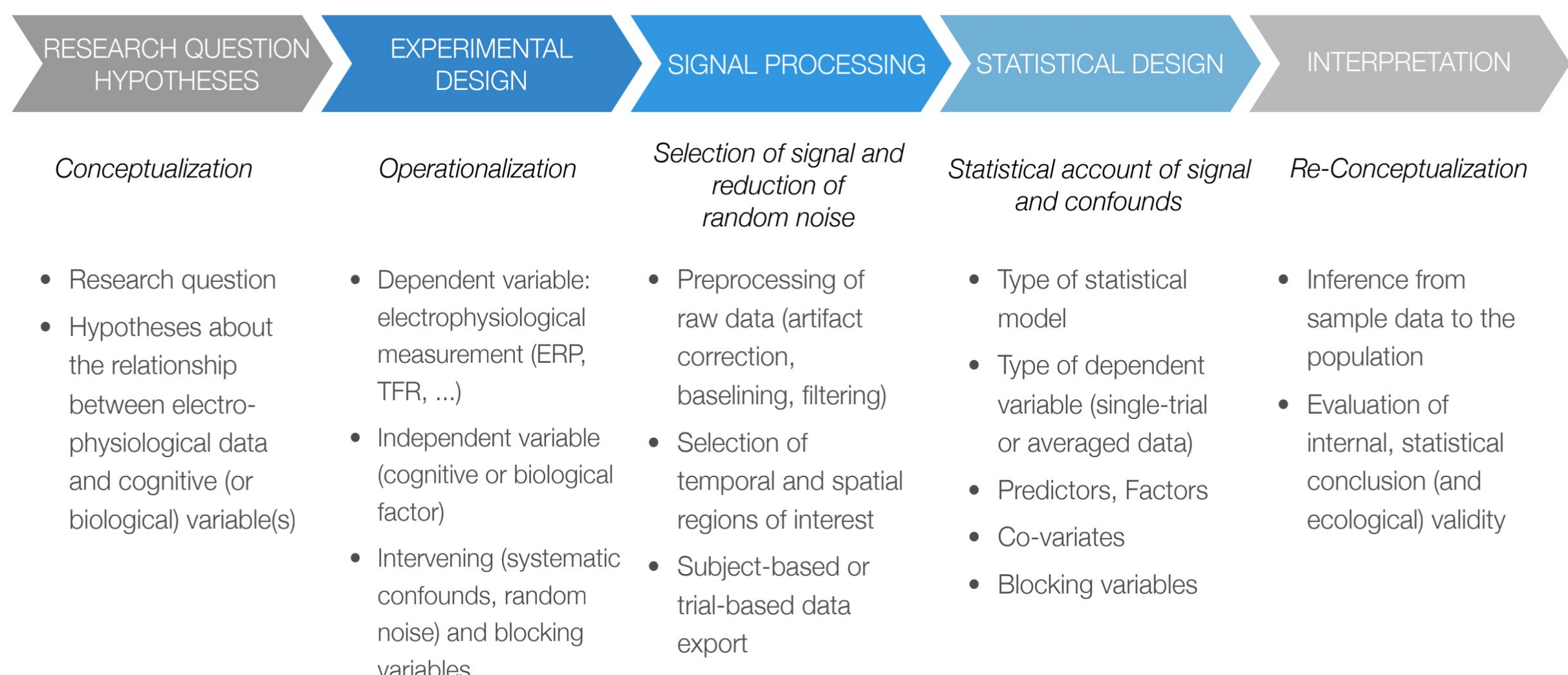




\section{Figure 2}

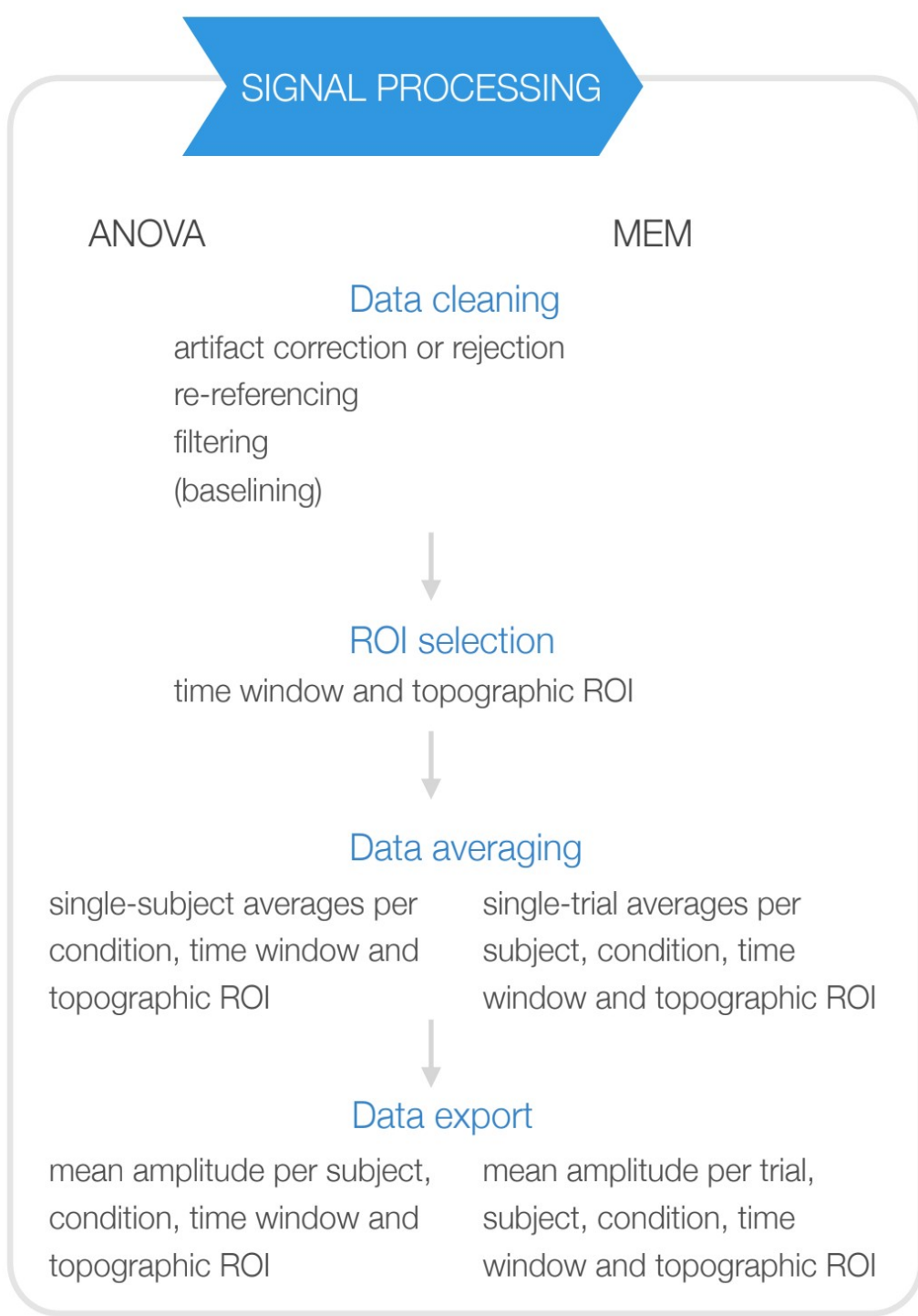

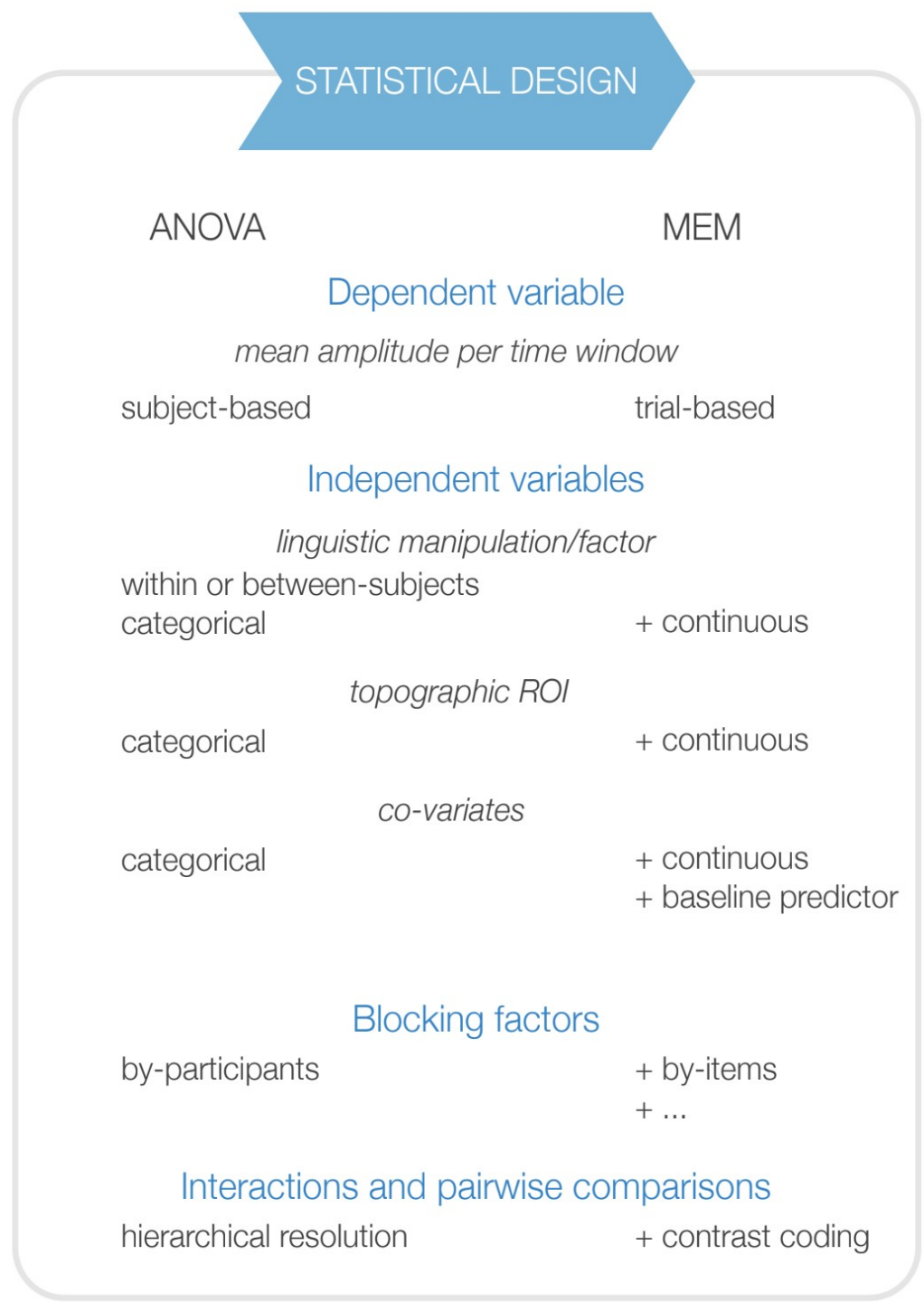

